\title{
Distribution Network Expansion Planning Based on Multi-objective PSO Algorithm
}

\author{
Chunyu Zhang, Yi Ding, Qiuwei Wu, Qi Wang, Jacob Østergaard \\ Center for Electric Power and Energy, Technical University of Denmark, Copenhagen, Denmark \\ Email: alex.316@live.com
}

Received January, 2013

\begin{abstract}
This paper presents a novel approach for electrical distribution network expansion planning using multi-objective particle swarm optimization (PSO). The optimization objectives are: investment and operation cost, energy losses cost, and power congestion cost. A two-phase multi-objective PSO algorithm is employed to solve this optimization problem, which can accelerate the convergence and guarantee the diversity of Pareto-optimal front set as well. The feasibility and effectiveness of both the proposed multi-objective planning approach and the improved multi-objective PSO have been verified by the 18-node typical system.
\end{abstract}

Keywords: Distribution Network Expansion Planning; Two-phase; Multi-objective PSO

\section{Introduction}

The restructure and deregulation of the global power industry have introduced fundamental changes to the practices of power system planning. Conventional single optimization objective approach such as the minimization of the investment cost is no longer suitable for the new market operation environment. The multi-objective planning approach has become necessary in order to take into account new problems caused by the competitive power market environment [1,2].

Over the past decade, a large amount of researches and literatures have been accomplished in multi-objective distribution network expansion planning approach. A detailed review of different methods can be found in $[3,4]$.

The distribution system planning problem dimension increases with number of nodes. Normally, numerical optimization tools such as nonlinear programming (NLP) [5] and dynamic programming [6] have been used to solve this problem with lower node systems. In multiobjective problems, there are some specific disadvantages in using these analytical solution strategies, i.e., curse of dimensionality, non-differentiability, discontinuous objective space etc [7]. Moreover, to get a set of solutions (as with Pareto-optimality principle), any numerical method requires several trial runs.

Considering the complex solution space, non-convex and nonlinear mixed integer objective functions, the solution of multi-objective distribution network planning problem is difficult to gain by many traditional optimization me- thods. Therefore, several intelligent algorithms have been used to enhance the performance of distribution expansion planning process, including greedy algorithms, genetic algorithms, particle swarm optimization, and evolutionary optimization. Particle swarm optimization (PSO) is one of the most widely used multi-point search algorithms using stochastic behavior. PSO is developed by inspiring with social behavior that is observed in nature such as flocks of birds and schools of fish [8].

In this paper, a distribution network expansion planning approach is proposed to optimize three objectives: the investment and operation cost, energy losses cost, and power congestion cost. Accordingly, a two- phase multi-objective PSO is introduced, which accelerates the convergence and guarantees the diversity of Pareto- optimal front set as well. Case study based on the 18- node system is conducted to demonstrate the feasibility and effectiveness of both the proposed planning approach and the improved multi-objective PSO algorithm.

\section{Problem Formulation}

\subsection{Multi-Objective Planning Model}

The power system restructuring forces a change in duties and objectives of traditional planning and it compels to consider several objectives that are in mutual conflict. The proposed planning model minimizes different cost functions related to the cost of investment and operation $s_{1}(\mathbf{x})$, cost of energy losses $s_{2}(\mathbf{x})$ and cost of power congestion $s_{3}(\mathbf{x})$ in the form of multi-objective optimization (1). 


$$
\begin{aligned}
& \text { s.t. } \\
& P_{D G, i j}^{\min } \leq P_{D G, i j} \leq P_{D G, i j}^{\max } \\
& Q_{D G, i j}^{\min } \leq Q_{D G, i j} \leq Q_{D G, i j}^{\max } \\
& P_{D G, i j}^{t+1}-P_{D G, i j}^{t} \leq \text { Ramp }^{\uparrow} \\
& P_{D G, i j}^{t}-P_{D G, i j}^{t+1} \leq R^{\prime} p^{\downarrow} \\
& V_{m}^{\min } \leq V_{m} \leq V_{m}^{\max } \\
& -P_{m n}^{\min } \leq P_{m n} \leq P_{m n}^{\max }
\end{aligned}
$$$$
\min S(\mathbf{X})=\left\{s_{1}(\mathbf{X}), s_{2}(\mathbf{X}), s_{3}(\mathbf{X})\right\}
$$

where $P_{D G, i j} / Q_{D G, i j}$ is the active power and reactive power of $j$ th technology of DG-unit at bus $i$. Constrains $(1 b)$ and $(1 c)$ impose the DG-unit production in a specific range from $P_{D G, i j}^{\min } / Q_{D G, i j}^{\min }$ to $P_{D G, i j}^{\max } / Q_{D G, i j}^{\max }$. Constrains (1e) and (1f) indicate The ramp up and ramp down limitations are expressed as $\operatorname{Ramp}^{\uparrow}$ and Ramp ${ }^{\downarrow}$, which are indicated for each DG at each time period $t$, it could be minutes or hours. The voltages $V_{m}$ limitation at each distribution node $m$ is caped with constraints (1f). Meanwhile, the distribution line capacity and power flow capacity limitation of distribution feeder $m n$ is limited by constraints $(1 g)$. Here, the indices of $m, n$ and $i$ are all for the bus number of this distribution network.

\subsection{Investment and Operation Cost}

The total cost function $\left(s_{1}\right)$ for investment and operation of DG units is given in (2). The costs of installing DGs in candidate buses of a distribution network are considered as follow. It is assumed that DG units can be installed in all load buses; however, the best sites are determined according to the optimization process. Yearly investment cost of each technology is determined based on discount rate and payment period according to (3). As an incentive program for renewable sources, this parameter is calculated using a low discount value. Besides, feed-in tariffs increase the capacity factor o of DG technologies.

$$
\begin{gathered}
S_{1}=\sum_{i=1}^{N} \sum_{j \in \Omega} I_{C j} \cdot P_{D G, i j}+\sum_{i=1}^{N} \sum_{j \in \Omega} O_{C j} \cdot P_{D G, i j} \cdot A_{j} \cdot a_{j} \cdot T \\
I_{C j}=\frac{d^{t}(1-d)}{1-d^{t}} \cdot T_{I C j}
\end{gathered}
$$

where $I_{C j}$ and $O_{C j}$ are the yearly investment and hourly operating costs of jth technology of DG-unit, respectively, $T_{I C j}$ is the total investment cost of $j$ th DG technology, $d$ and $t$ are, respectively, discount rate and payment period, $N$ is the number of load buses in the distribution system; $T$ is yearly operating hours, $A j$ is the availability factor related to $j$ th technology; and $a_{j}$ is the average capacity factor of $j$ th DG technology considering incentive effects of feed-in tariff policy.

\subsection{Energy Losses Cost}

This objective function $\left(s_{2}\right)$ attempts to minimize the total cost of the energy losses in the distribution network due to installation of DG units. This function is strongly related to the locations of DG units in the distribution system. The power flow in the feeder connecting buses $i$ and $j$ is used to formulate the energy loss function as follows:

$$
\begin{gathered}
S_{2}=\sum_{i=1}^{N} \sum_{j=i+1}^{N} \frac{\left(\left|V_{i}\right|-\left|V_{j}\right|\right)^{2}}{\left|Z_{i j}\right|} P_{f} \cdot L_{f} \cdot k \cdot T \\
P_{i j} \doteq\left|V_{i}\right| \cdot \frac{\left(\left|V_{i}\right|-\left|V_{j}\right|\right)}{\left|Z_{i j}\right|} \cdot P_{f}
\end{gathered}
$$

where $N$ is the total number of buses in the distribution system; $V$ is the bus voltage, $Z_{i j}$ and $P_{i j}$ are the impedance and power flow of branch $i-j$, respectively; $P_{f}$ is the system power factor and $k$ is the expected price of electricity.

\subsection{Power Congestion Cost}

The congestion cost $\left(s_{3}\right)$ is given by

$$
s_{3}=\sum_{(i, j) \in \Omega} P_{i j}\left(l_{j}-l_{i}\right)+P_{f} \cdot W
$$

where $l_{i}, l_{j}$ is the locational marginal price at bus $i, j$, which are the Lagrange multipliers or shadow prices of the power flow constraints. $P_{f}$ is the large penalty factor. $W$ is the total artificial generator (shed load) in normal operation conditions.

\section{Two-phase Multi-objective PSO}

\subsection{PSO}

Recent years, PSO has been drawn more and more attention, due to its powerful searching ability in function optimization. In the searching space, the behavior of a particle can be recognized as the velocity $(v)$ and position $(x)$. The updating rule of PSO will steer the particle swarm to gather in more promising area with better objective value. For the ith particle in iteration $t$ the behavior of each particle can be expressed as

$$
\begin{gathered}
v_{i j}^{t+1}=w \cdot v_{i j}^{t}+c_{1} \cdot r_{1} \cdot\left(p_{i j}^{t}-x_{i j}^{t}\right)+c_{2} \cdot r_{2} \cdot\left(p_{g i}^{t}-x_{i j}^{t}\right) \\
x_{i j}^{t+1}=x_{i j}^{t}+v_{i j}^{t}
\end{gathered}
$$

where $X_{i}=\left(x_{\mathrm{i} 1}, X_{\mathrm{i} 2}, \ldots, X_{\mathrm{in}}\right)$ represents the position and $V_{i}=\left(v_{\mathrm{i} 1}, \mathrm{v}_{\mathrm{i} 2}, \ldots, v_{\mathrm{in}}\right)$ denotes the velocity, and the local best position can be written as $p_{\mathrm{i}}=\left(p_{\mathrm{i} 1}, p_{\mathrm{i} 2}, \ldots, p_{\mathrm{in}}\right)$. The global best position (guide) among all the particles can be given as $p_{\mathrm{g}}=\left(p_{\mathrm{g} 1}, p_{\mathrm{g} 2}, \ldots, p_{\mathrm{gn}}\right) . w$ is the inertia weight factor, $c_{1}$ 
and $c_{2}$ are non-negative constants, and $r_{1}$ and $r_{2}$ are random numbers located in $[0,1]$.

Currently, multi-objective PSO is becoming much hotter for the multi-objective optimization problems. The major differences between single objective PSO and multi-objective PSO are the proper introduction of archive to reserve Pareto-optimal candidates and the appropriate selection of guide particles for multi-objective optimization [9]. Guide particle is the global best position among all the particles proceeded in the PSO algorithm.

As shown in the literature, Mostaghim and Teich [10] proposed the Sigma method first in 2003, which selected the best guides for each particle mainly focusing on improving the convergence to the Pareto front. However, this approach could not gain the good convergence and uniform diversity simultaneously. Furthermore, Coello and Lechgua [11] introduced the global guide selection method based on Pareto optimality and hypercube in the objective function space to maintain the diversity of the particles. However, its convergence rate is quite low [12]. The performance of multi-objective PSO optimization algorithm needs to be improved.

\subsection{Two-phase Multi-objective PSO}

1) The steps of two-phase multi-objective PSO.

Step 1: Initialize the parameters of two-phase multiobjective PSO.

Step 2: Initialize the position and velocity of each particle in set $P$ and archive $A$. Set the initial position as the individual best position $p_{i j}^{t}=X_{i}$ of each particle;

Step 3: For iteration $t=1$ to $T$,

a) Select the global best position $p_{g i}^{t}$ for each particle from the archive $A$,

c) Update position and velocity of every particle refer to the (7)-(8),

d) If $t<0.8 T$, the particle is mutated according to the archive set,

e) Update the local best position $p_{i j}^{t}$, if the current location is dominated by its local best location $p_{i}$, then the previous location is maintained,

f) Repeat step 3 for next iteration.

2) Strategy of mutation.

As discussed in [14], a mutation operator is employed in this Multi-objective PSO considering this algorithm may converge to local optimal fronts.

Here, mutation probability $\left(P_{m}\right)$ is reduced with the iteration of the algorithm according to

$$
p_{m}=1-\frac{C_{g}}{Z}
$$

where $C_{g}$ is the number of current generation. For each particle, the variable $m_{r}$ is a random number in the range of $[0,1]$. If $m_{r}<P_{g}$, the particle is randomly selected for mutation according to

$$
x_{i}=\theta \cdot \mu \cdot\left(1-m_{r}\right) \cdot v_{i}+x_{i-1}
$$

where $\mu$ points out the direction in mutation and $\theta$ controls the distance covered by a jump. In this paper, $\mu=3$ and $\theta$ is set as \pm 1 randomly. If the solution is beyond its boundary by mutation, it is moved to the corresponding boundary.

\subsection{Program Flow}

The major multi-objective distribution network problem modules and the general flow of the program are shown in Figure 1. The Fuzzy satisfying decision making approach [7] is introduced in this program.

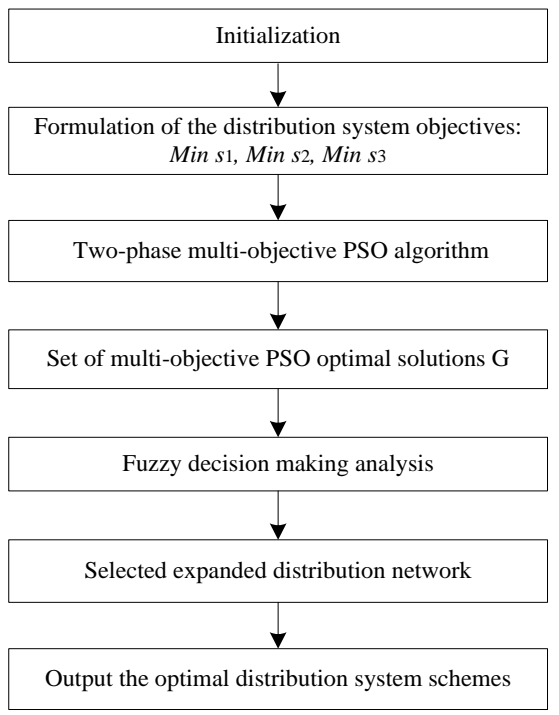

Figure 1. Mmulti-objective distribution network flow.

\section{Case Study}

Case study has been carried out on the 18-node system, to prove the effectiveness of both the proposed multiobjective planning approach and the introduced two-phase multi-objective PSO solver. The parameter details of the 18-node system can be found in [13]. The system has 28 right-of-ways, the active power transmission limit of each line is 50MW, and line cost is $\$ 130,500 / \mathrm{km}$.

In this section, the best three planning schemes of the corresponding 18-node system are presented in detail in Figure 2 and Table 1, which all indicate the network of the 18-node system has a relative tightly linked structure. Further comparison between $\mathrm{M}_{1}$ and $\mathrm{M}_{2}$ shows that, under the almost same total cost, $\mathrm{M}_{2}$ is definitely better than $M_{1}$ due to its lower energy losses cost and power congestion cost, with strong future adaptability. Compared with $\mathrm{M}_{3}, \mathrm{M}_{2}$ has extremely high power congestion cost and obviously double energy losses cost, but $\mathrm{M}_{3}$ with a notable increase on total cost. 


\section{Conclusion}

A multi-objective distribution network expansion approach is proposed, the objectives are the investment and operation cost, energy losses cost, and power congestion cost. And the solving algorithm based on the two-phase multi-objective PSO is also introduced in this paper.
The planning results of the 18-node system show that, for a practical system, the proposed multi-objective distribution network expansion method can effectively enhance the distribution capacity by adding specific new lines under the variety conditions of future uncertainties.

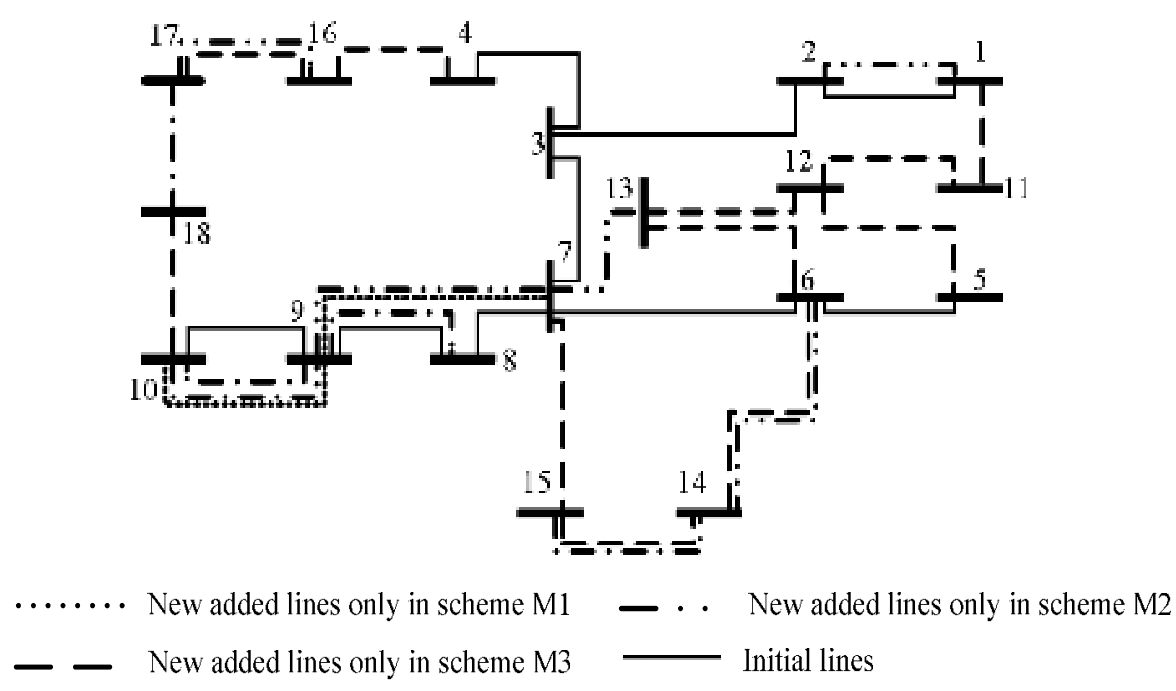

Figure 2. Topology of the expanded network of the 18-node system.

Table 1. Best planning schemes of the 18-node system.

\begin{tabular}{cccc}
\hline Schemes & $\mathrm{M}_{1}$ & $\mathrm{M}_{2}$ & $\mathrm{M}_{3}$ \\
\hline New added distribution network lines & $7-9,9-10$ & $8-9,9-10(2), 1-2,6-14,7-9$, & $1-11,4-16,5-12,6-13,6-14,7-15$, \\
& & $7-13,16-17,14-15$ & $10-18,11-12,12-13,14-15,16-17,17-18$ \\
Investment and operation cost $\left(\times 10^{3} \$\right)$ & 2043.01 & 10452.73 & 12250.56 \\
Energy losses cost $\left(\times 10^{3} \$\right)$ & 5583.37 & 572.25 & 282.45 \\
Power congestion cost $\left(\times 10^{3} \$\right)$ & 2832.43 & 252.40 & 34.66 \\
Total cost $\left(\times 10^{3} \$\right)$ & 10458.81 & 10397.38 & 12837.67 \\
\hline
\end{tabular}

Considering efficiency, reliability, and economic, the best planning schemes can be put forward by the two-phase multi-objective PSO, which shows its superiority as well.

Further research can focus on the multi-stage and multi-objective model, which should consider the uncertainty of the bidding parameters and other uncertain factors in distribution expansion problem.

\section{Acknowledgements}

The authors gratefully acknowledge the financial supports and the strategic platform for innovation \& research provided by Danish national project iPower.

\section{REFERENCES}

[1] A. Barin, L. F. Pozzatti, L. N. Canha, et al., "Multi-objective Analysis of Impacts of Distributed Generation Placement on the Operational Characteristics of Networks for Distribution System Planning," International Journal of Electrical Power \& Energy Systems, Vol. 32, 2010, pp. 1157-1164. doi:10.1016/j.ijepes.2010.06.015

[2] Z. Kai, A. P. Agalgaonkar, K. M. Muttaqi and S. Perera, "Multi-objective Optimization for Distribution System Planning with Renewable Energy Resources," in Proc. 2010 IEEE International Energy Conference, pp. 670-675.

[3] A. H. Mantway and M. M. Al-Muhaini, "Multi-objective BPSO Algorithm for Distribution System Expansion Planning Including Distributed Generation," in Proc. 2008 IEEE International Transmission \& Distribution, pp. 134-141.

[4] R. Rosado and D. Navarro, "Possibilistic Model Based on Fuzzy Sets for the Multiobjective Optimal Planning of Electric Power Distribution Networks," IEEE Trans. Power Systems, Vol. 19, 2004, pp. 1801-1810. doi:10.1109/TPWRS.2004.835678

[5] A. Soroudi and M. Ehsan, "A Distribution Network Expansion Planning Model Considering Distributed Genera- 
tion Options and Techo-Economical Issues,” International Journal of Energy, Vol. 35, 2010, pp. 3364-3374.

[6] E. G. Carrano, F. G. Guimaraes, R. H. C. Takahashi, et al., "Electric Distribution Network Expansion Under Load-Evolution Uncertainty Using an Immune System Inspired Algorithm," IEEE Trans. Power Systems, Vol. 22, 2007, pp. 851-861.

[7] T. S. Chung, K. K. Lee, G. J. Chen, J. D. Xie and G. Q. Tang, "Multi-Objective Transmission Network Planning by a Hybrid GA Approach with Fuzzy Decision Analysis,” International Journal of Electrical Power \& Energy Systems, Vol. 25, 2003, pp. 187-192.

[8] R. Poli, J. Kennedy and T. Blackwell, Particle swarm optimization an overview, Vol. I. MIT: Wiley, 2010, p. 2.

[9] A. M. R. Sierra and C. A. Coello, "Multi-objective Particle Swarm Optimizers: A Survey of the State-of-the-art,” International Journal of Computational Intelligence Research, Vol. 3, 2006, pp. 287-308.

[10] S. Mostaghim and J. Teich, "Strategies for finding good local guides in multi-objective particle swarm optimization (MOPSO),” in Proc. 2003 IEEE Swarm Intelligence Symposium, pp. 26-33.

[11] M. S. Lechuga, and C. A. Coello, "Handling Multiple Objectives with Particle Swarm Optimization," IEEE Trans. Evolutionary Computation, Vol. 3, 2006, pp. 256-279.

[12] N. C. Sahoo, S. Ganguly and D. Das, "Fuzzy-Pareto-dominance Driven Possibilistic Model Based Planning of Electrical Distribution Systems Using Multi-objective Particle Swarm Optimization,” Expert Systems with Applications, Vol. 39, 2012, pp. 881-893. doi:10.1016/j.eswa.2011.07.086

[13] X. Wang and J. R. McDonald, Modern Power System Planning, McGraw-Hill Book Company, London, 1993.

[14] G. Hu, Z. Mao, and D. He, "Multi-objective optimization for leaching process using improved two-stage guide PSO algorithm," J. Cent. South Univ. Technol., vol. 18, pp. 1200-1210, 2011. 